\title{
Inquérito Luso-brasileiro de Nutrição Oncológica do Idoso: um Estudo Multicêntrico
}

\author{
Lusa-Brazilian Survey of the Old-aged Oncology Nutrition: a Multicenter Study \\ Encuesta Luso-Brasilena de Nutrición Oncológica de los Ancianos: un Estudio \\ Multicéntrico
}

\author{
INSTITUTO NACIONAL DE CÂNCER JOSÉ ALENCAR GOMES DA SILVA. Inquéri- \\ to Luso-brasileiro de Nutrição Oncológica do Idoso: um estudo multicêntrico. Rio de \\ Janeiro: INCA, 2015. 76p. \\ ISBN: 978-85-7318-270-5 (versão impressa)/ 978-85-7318-271-2 (versão eletrônica)
}

Taís Facina ${ }^{1}$

Organizado por Nivaldo Barroso de Pinho, nutricionista do Hospital do Câncer I/Instituto Nacional de Câncer José Alencar Gomes da Silva (INCA), este livro contou com a participação de uma equipe multidisciplinar e de diversas instituiçóes do Brasil e do mundo. Baseado em um estudo multicêntrico e multirregional, no qual foi possível caracterizar a desnutrição do paciente oncológico intra-hospitalar no Brasil no momento da internação, o livro mostra a prevalência da desnutrição em pacientes idosos e como o uso de ferramentas adequadas para a triagem nutricional pode ser útil para uma intervenção nutricional mais precisa, evitando o aumento da morbimortalidade e do tempo de internaçáo.

A técnica utilizada para a triagem nutricional deve ser simples e rápida, facilmente incorporada à rotina diária de avaliação dos pacientes nos diversos ambientes ambulatoriais e hospitalares. Atualmente, os instrumentos mais conhecidos são o Must (do inglês malnutrition universal screening/instrumento universal para triagem da desnutrição), NRS (do inglês malnutrition risk screening/triagem de risco nutricional) e MAN (do inglês mini nutritional assessment/ miniavaliação nutricional). Porém, para atender às particularidades dos pacientes com câncer, é muito usado um instrumento chamado Avaliação Subjetiva Global Produzida pelo Próprio Paciente (ASG-PPP).

Neste estudo multicêntrico, descritivo e prospectivo foram incluídos todos os pacientes de ambos os sexos, idosos (acima de 65 anos), portadores de tumores malignos, independentemente de localização ou estadiamento da doença, internados nas instituiçóes brasileiras e portuguesas que participaram do projeto, durante o período de setembro a outubro de 2014. Os pacientes foram avaliados nas primeiras 48 horas após a internação.

Foram 44 instituiçôes hospitalares de todo o Brasil, totalizando 3.062 idosos, e seis de Portugal, com 195 idosos. Desse total, mais da metade relataram perdas de peso nos últimos três meses, sendo que mais do que $34 \%$ com perdas superiores a $3 \mathrm{~kg}$. A conclusão do estudo mostrou também que o percentual de pacientes que permaneceram mais tempo internados (mais de 30 dias de internação) foi maior naqueles com história de perda de peso maior que $3 \mathrm{~kg}$. Sendo assim, a história de perda de peso deve ser considerada como preditivo de maior tempo de internaçáo.

Além do tempo de internação, outros parâmetros são utilizados, como o índice de massa corporal (IMC), nível de independência da populaçáo idosa, e circunferência de panturrilha (CP). A presença de risco nutricional e de desnutrição nessa população idosa também determinou um tempo maior de internação. Em indivíduos com maior tempo de internação, foi encontrada uma prevalência maior de desnutrição e de risco nutricional, quando comparados com indivíduos com tempo de internaçáo inferior a 30 dias $(85,5 \%$ versus $70,8 \%)$.

1Jornalista. Pós-graduada em "Produçâo do Livro”. Editora de Publicaçōes Científicas no Instituto Nacional de Câncer José Alencar Gomes da Silva (INCA). 
A partir desses dados sobre o perfil nutricional dos pacientes idosos oncológicos, obtidos pela triagem nutricional apresentada no estudo em questão, o livro faz uma previsão de desfecho, como tempo e taxa de mortalidade durante a internaçáo, auxiliando o profissional a intervir, de forma precoce, utilizando estratégias que possam prevenir morbidade e mortalidade intra-hospitalares. 